University of Wollongong

Research Online

Faculty of Engineering and Information

Faculty of Engineering and Information

Sciences - Papers: Part A

Sciences

$1-1-2010$

Investigation of high resolution compact gamma camera module based on a continuous scintillation crystal using a novel charge division readout method

Qiu-Sheng Dai

Chinese Academy Of Sciences

Cui-Lan Zhao

Chinese Academy Of Sciences

Hualin Zhang

Ohio State University

Yujin Qi

Chinese Academy Of Sciences, yujin@uow.edu.au

Follow this and additional works at: https://ro.uow.edu.au/eispapers

Part of the Engineering Commons, and the Science and Technology Studies Commons

Research Online is the open access institutional repository for the University of Wollongong. For further information contact the UOW Library: research-pubs@uow.edu.au 


\title{
Investigation of high resolution compact gamma camera module based on a continuous scintillation crystal using a novel charge division readout method
}

\begin{abstract}
The objective of this study is to investigate a high performance and lower cost compact gamma camera module for a multi-head small animal SPECT system. A compact camera module was developed using a thin Lutetium Oxyorthosilicate (LSO) scintillation crystal slice coupled to a Hamamatsu H8500 position sensitive photomultiplier tube (PSPMT). A two-stage charge division readout board based on a novel subtractive resistive readout with a truncated center-of-gravity (TCOG) positioning method was developed for the camera. The performance of the camera was evaluated using a flood $99 \mathrm{mTc}$ source with a fourquadrant bar-mask phantom. The preliminary experimental results show that the image shrinkage problem associated with the conventional resistive readout can be effectively overcome by the novel subtractive resistive readout with an appropriate fraction subtraction factor. The response output area (ROA) of the camera shown in the flood image was improved up to $34 \%$, and an intrinsic spatial resolution better than $2 \mathrm{~mm}$ of detector was achieved. In conclusion, the utilization of a continuous scintillation crystal and a flat-panel PSPMT equipped with a novel subtractive resistive readout is a feasible approach for developing a high performance and lower cost compact gamma camera.
\end{abstract}

\section{Keywords}

continuous, scintillation, crystal, novel, investigation, charge, high, division, readout, method, resolution, compact, gamma, camera, module

\author{
Disciplines \\ Engineering | Science and Technology Studies
}

\section{Publication Details}

Dai, Q., Zhao, C., Zhang, H. \& Qi, Y. (2010). Investigation of high resolution compact gamma camera module based on a continuous scintillation crystal using a novel charge division readout method. Chinese Physics C, 34 (8), 1148-1152. 
Investigation of high resolution compact gamma camera module based on continuous scintillation crystal using novel charge division readout method*

\author{
DAI Qiu-Sheng ${ }^{1}$, ZHAO Cui-Lan ${ }^{1}$, Zhang Hualin² $^{2}$, QI Yu-Jin ${ }^{1+}$ \\ ${ }^{1}$ Shanghai Institute of Applied Physics, Chinese Academy of Sciences, Shanghai 201800, China \\ ${ }^{2}$ Department of Radiation Medicine, the Ohio State University, Columbus, OH 43210, USA
}

\begin{abstract}
The objective of this study is to investigate high performance and lower cost compact gamma camera module for multi-head small animal SPECT system. A compact camera module was developed using a thin Lutetium Oxyorthosilicate (LSO) scintillation crystal slice coupled to a Hamamatsu H8500 position sensitive photomultiplier tube (PSPMT). A two-stage charge division readout board based on novel subtractive resistive readout with truncated center-of-gravity (TCOG) positioning method was developed for the camera. The performance of the camera was evaluated using a flood ${ }^{99 \mathrm{~m}} \mathrm{Tc}$ source with four-quadrant bar-mask phantom. The preliminary experimental results show that the image shrinkage problem associated with the conventional resistive readout can be effectively overcome by the novel subtractive resistive readout with an appropriate fraction subtraction factor, the response output area (ROA) of camera shown in the flood image was improved up to $34 \%$, and an intrinsic spatial resolution better than $2 \mathrm{~mm}$ of detector was achieved. In conclusion, the utilization of continuous scintillation crystal and flat-panel PSPMT equipped with the novel subtractive resistive readout is a feasible approach for development of high performance and lower cost compact gamma camera.
\end{abstract}

Key words compact gamma camera module, truncated center-of-gravity method, image shrinkage effect

PACS 87.57.cf, 87.57.ue, 85.60.Ha

\title{
1 Introduction
}

Single photon emission computed tomography (SPECT) imaging plays an important role in the rapidly growing field of molecular imaging ${ }^{[1,2]}$. Recent advance of SPECT has been focused on development of multi-camera SPECT system for small animal imaging with large detection efficiency while maintaining high spatial resolution ${ }^{[3-5]}$. The multi-camera SPECT system usually requires high-resolution compact gamma camera module.

The main approach for the high-resolution compact gamma camera module is based on the discrete scintillation crystal array with position sensitive photomultiplier tube (PSPMT). It has been well-proven in obtaining high spatial resolution, good performance and reliability in breast and small-animal imagers ${ }^{[6-12]}$. However, the high price of the discrete crystal array as compared to the continuous crystal usually leads to expensive cost for multi-camera SPECT system. So a lower cost alternative to the high resolution discrete crystal design using continuous crystal slab with PSPMT for compact modular camera has gained more and more interest ${ }^{[13-15]}$.

The continuous scintillation crystal slab with cross-anode PSPMT for development of

\footnotetext{
* Supported by National Natural Science Foundation of China $(30570520,10875162)$

E-mail: qiyujin@sinap.ac.cn
} 
miniature gamma camera has been studied by several groups ${ }^{[14-15]}$. Their results show that the performance of those miniature gamma cameras was limited by the conventional resistive readout with the Anger positioning method ${ }^{[16]}$, which resulted in significant shrinkage of the effective field-of-view (FOV) of the camera ${ }^{[15]}$. Therefore, the improvement of the readout and positioning method is essential for development of high performance and lower cost modular gamma camera.

The aim of our study is to develop high performance and lower cost compact gamma camera module for multi-camera small animal SPECT system. In this study, we investigated a compact gamma camera module using a continuous lutetium oxyorthosilicate (LSO) scintillation crystal slab coupled to a compact multi-anode flat panel PSPMT (Hamamatsu H8500). A novel subtractive resistive readout based on truncated center-of-gravity (TCOG) positioning method was developed for the camera ${ }^{[7,17]}$. The performance of the detector module was evaluated by experiments.

\section{Readout methods}

\subsection{Conventional readout}

Two main readout schemes have been adopted for the compact gamma camera based on PSPMT. One is based on channel-by-channel readout method, which requires complex readout processing circuits and the data acquisition system because the individual anode signals need to be processed for decoding the position information. The other is based on charge division method using resistive matrix network, which needs simple readout circuits and data processing. So the resistive charge division method has been widely used in the development of compact gamma camera.

In the charge division method, the anodes are directly connected to a simple resistive network which provides a proportional charge division as function of position defined by the anode location, generally with four output channels. The coordinate of charge distribution centroid is determined by the following equation ${ }^{[18]}$ :

$$
x=\frac{\sum w_{i}^{X} q_{i}^{X}}{\sum q_{i}^{X}}, \quad y=\frac{\sum w_{i}^{Y} q_{i}^{Y}}{\sum q_{i}^{Y}}
$$

where $x$ and $y$ are the event coordinates, $w_{i}$ is the weighting factor for the $i$ th strip anode, $q_{i}$ is the charge collected on it and the superscripts $\mathrm{X}$ and $\mathrm{Y}$ denote the anode direction.

The conventional resistive charge division method usually could reduce the readout channels down to four outputs. So the following signal processing and data acquisition become much simpler and lower cost. However, it has a major disadvantage in the position determination with serious non-linearity because of the significant contributions of noises and cross-talks far away from the incident area in the position calculations with equation (1), which results in a significant shrinkage of the effective field-of-view (FOV) of the camera image ${ }^{[15]}$.

\subsection{A novel subtractive resistive readout with TCOG positioning method}

In order to overcome the problems of the conventional resistive readout, a novel subtractive resistive readout method was proposed ${ }^{[17]}$, shown in Fig. 1 . It could be regarded as the combination of the individual readout with the conventional resistive readout. The basic idea of the subtractive resistive readout is to implement a fractional subtraction technique in the readout circuit to subtract the long tail of the charge distributions on the anode plane of the PSPMT and 
remove the noise contributions far from the gamma-ray incident area. Then a truncated center-of-gravity (TCOG) algorithm is applied to improve the accuracy of the position determination and to reduce the image shrinkage effect.

The fractional subtraction factor (referred as f-factor) is critical in the subtractive resistive readout since it determines the selection size of the local region which involves in the position calculations. Therefore, it is important to select an appropriate f-factor in subtractive resistive readout. If the f-factor is set too high, the uniformity of the flood image would become worse while if the f-factor is set too low, the nonlinearity and image shrinkage couldn't be suppressed. We usually determine the appropriate f-factor by experiments.

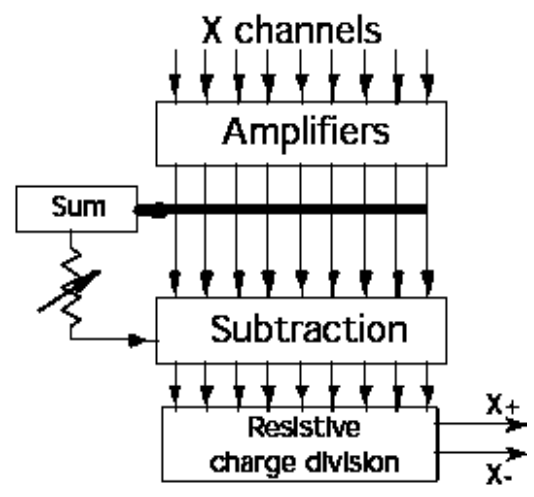

(a)

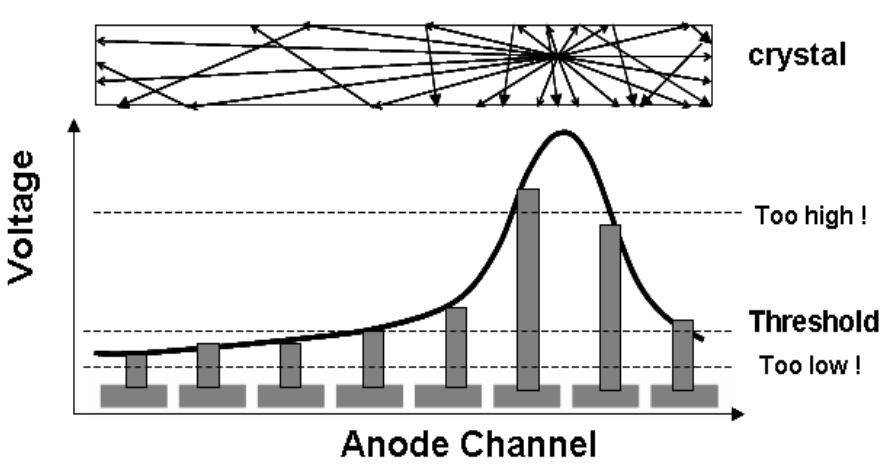

(b)

Fig. 1 (a) Diagram of the subtractive resistive readout ${ }^{[17]}$ and (b) a simple illustration of the truncated center-of-gravity positioning principle

\section{Compact gamma camera module}

The compact gamma camera module that we developed is based on a continuous LSO crystal slab with a Hamamatsu H8500 PSPMT, shown in Fig. 2. The LSO slab from the Shanghai SICCAS High Technology Corp. is $50 \mathrm{~mm}$ square with a thickness of $2 \mathrm{~mm}$, which has a stopping power of about $80 \%$ for gamma rays at the energy of $140 \mathrm{keV}$. The LSO crystal slab is directly coupled onto a H8500 PSPMT. The H8500 is a square, compact flat-panel PSPMT with an external dimensions of $52 \times 52 \times 28 \mathrm{~mm}^{3}$, a sensitive photocathode area of $49 \times 49 \mathrm{~mm}^{2}$, and a glass window thickness of $1.5 \mathrm{~mm}$. It has 12 metal channel dynodes with a gain of about $10^{6}$ and an $8 \times 8$ array of anode pads to collect the multiplied charges.

The readout circuit board, shown in Fig. 2(c), was developed using a novel two-stage charge division method. First, the incoming charge from 64 anodes is equally split into $\mathrm{X}$ and $\mathrm{Y}$ directions using a $2 \mathrm{D}$ symmetric decoupling resistive matrix ${ }^{[19]}$, which results in 8 -X and $8-\mathrm{Y}$ outputs. Then the 16 readout channels are further reduced to 4 outputs by using a subtractive resistive charge division ${ }^{[17]}$, where a fractional subtraction circuit was used to remove the long tail of charge distribution and the noise contributions far from the gamma-ray incident region. Then a truncated center-of-gravity (TCOG) algorithm is applied to determine the incident position of the detected gamma-rays. 


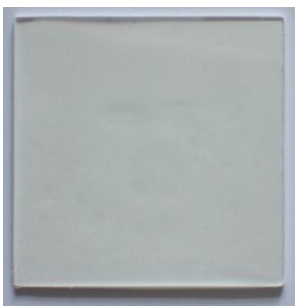

(a)

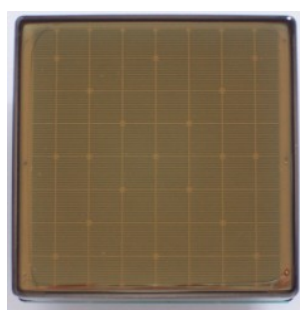

(b)

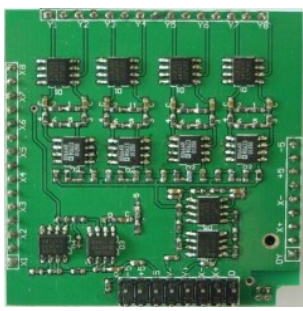

(c)

Fig. 2 Photograph of the components of our assembled detector module. (a) LSO crystal plate;

(b) H8500 PSPMT; (c) readout circuit board

\section{Experimental test methods}

The general performance of the assembled compact gamma camera module was evaluated using a flood ${ }^{99 \mathrm{~m}} \mathrm{Tc}$ source and the spatial resolution of the detector module was assed with a preliminary imaging experiment using a four-quadrant bar mask phantom, shown in Fig.3. In the flood imaging experiment, the detector performance was evaluated with different subtractive fraction factors of $0.1 \%, 3 \%$, and $6 \%$, in which the subtractive resistive readout with the f-factor of $0.1 \%$ is equivalent to the conventional resistive readout. The four-quadrant bar mask phantom, which has $180 \mathrm{~mm}$ in diameter, $5 \mathrm{~mm}$ in thickness, was made of lead strips which have widths equal to the spaces between them. The four bar patterns are $2 \mathrm{~mm}, 3 \mathrm{~mm}, 4 \mathrm{~mm}$, and $5 \mathrm{~mm}$. We only used the $2 \mathrm{~mm}$ bar-mask pattern in the imaging experiment. The flood images with different f-factors were used for the uniformity correction of the bar-mask phantom imaging.

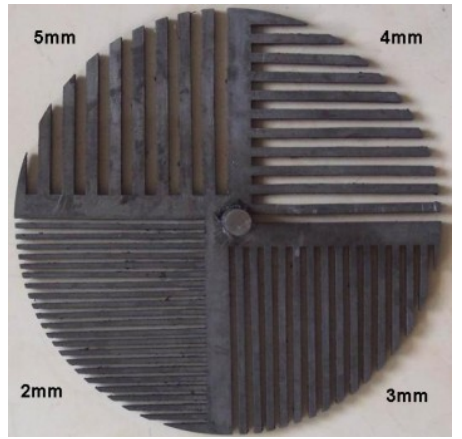

Fig. 3 Photograph of four-quadrant bar mask phantom

\section{Result and discussion}

The raw flood images acquired with different f-factors of $0.1 \%, 3 \%$, and $6 \%$ using a ${ }^{99 \mathrm{~m}} \mathrm{Tc}$ source are shown in Fig. 4. The results show that the image shrinkage effect of detector is very serious when the f-factor is set too low at $0.1 \%$ where the subtractive resistive readout is equivalent to the conventional resistive readout. When the f-factor is increased to an appropriate level around $6 \%$, the shrinkage of the flood image is significant reduced. 


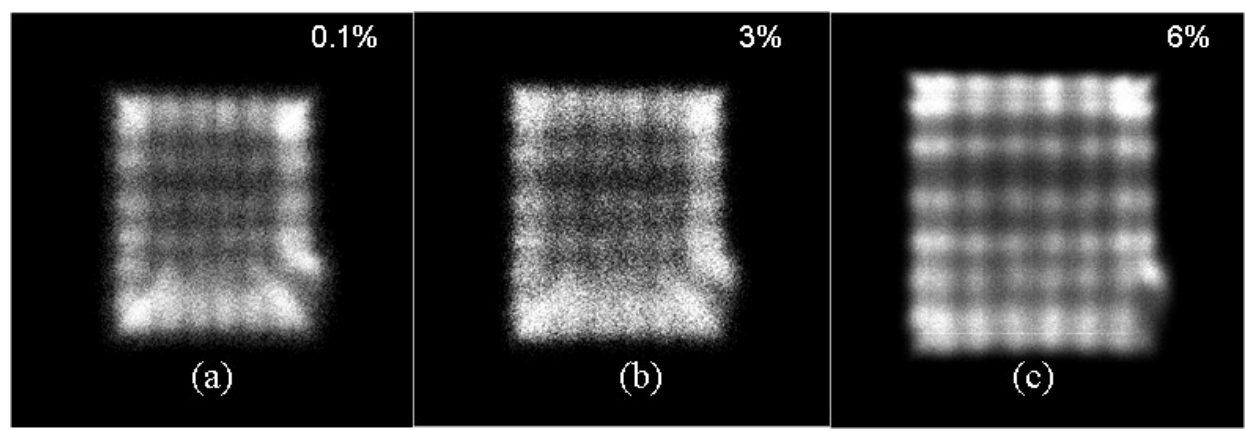

Fig. 4 Comparison of the raw flood images obtained with different f-factors using a flood ${ }^{99 \mathrm{~m}} \mathrm{Tc}$ source, all images are in the same scale.

The raw flood images acquired with the bar-mask are shown in Fig. 5(a,b,c) and the profiles of the flood images in the middle region are shown in Fig. 5(d). We calculate the response output area (ROA) of camera to qualitatively compare the image shrinkage effect with different f-factor settings. The results show that the ROA of the camera with f-factor of $6 \%$ almost reaches the active area of the PSPMT and it is increased about 34\% as compared to the ROA with f-factor of $0.1 \%$, which is equivalent with the conventional resistive charge division method. Therefore, the image shrinkage effect with the conventional resistive charge division is effectively suppressed by using the subtractive resistive charge division method.
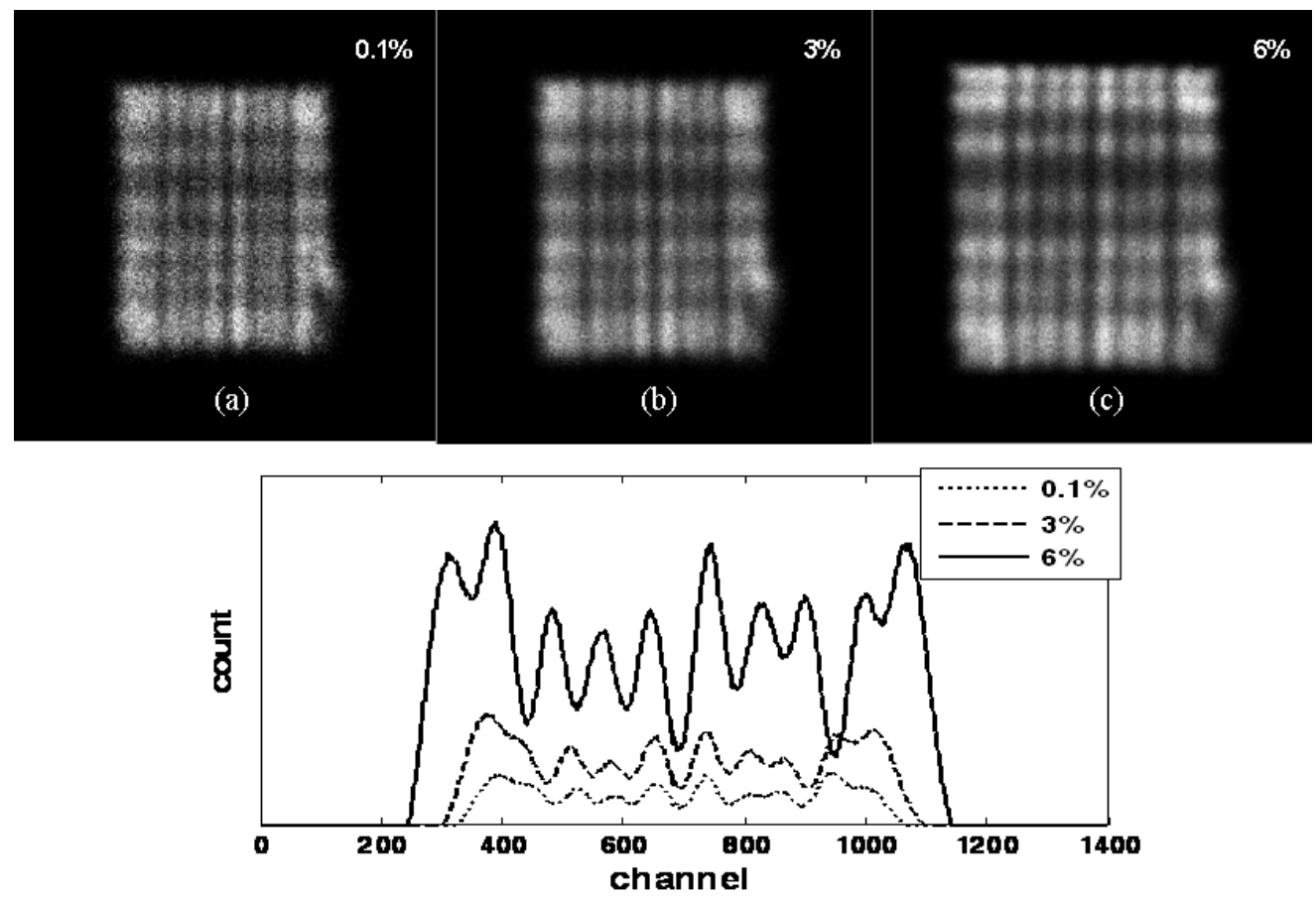

(d)

Fig. 5 Raw images obtained with bar mask, all images are in the same scale; the profiles of the flood images in the middle region are shown in the (d).

The bar image with f-factor of $6 \%$ using a coarse uniformity correction is shown in Fig. 6. The coarse uniformity correction was simply divided the raw bar image in Fig. 5(c) by the raw flood image in Fig. 4(c). We can see from Fig. 6 that the image quality is improved and the bar pattern with $2 \mathrm{~mm}$ separation is clearly resolved. This indicates that the compact gamma camera 
module that we designed could achieve an intrinsic spatial resolution under $2 \mathrm{~mm}$.
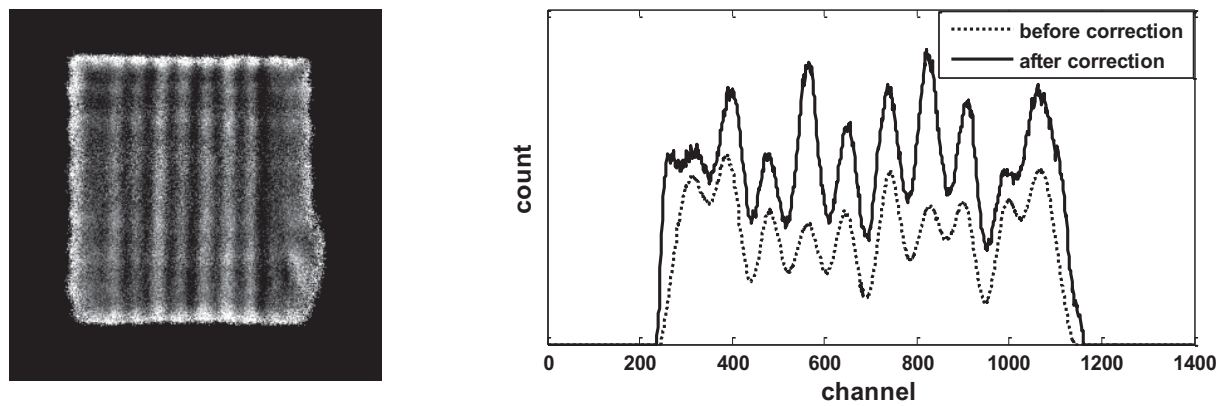

Fig. 6 Uniformity corrected image of the bar mask and the profile

\section{Conclusions}

We have developed a lower cost compact gamma camera module using a continuous LSO scintillation crystal slab and a Hamamatsu H8500 flat-panel PSPMT. A compact readout board based on a novel two-stage charge division method was developed for the camera. With the novel subtractive resistive readout and TCOG positioning algorithm, the image shrinkage effect associated with the conventional resistive readout can be effectively overcome to achieve high performance of the camera. In conclusion, the utilization of continuous scintillation crystal and flat-panel PSPMT equipped with the novel subtractive resistive readout is a feasible approach for development of high performance and lower cost compact gamma camera.

\section{References}

[1] Meikle SR, Kench P, Kassiou M, et al. Phys Med Biol. 2005, 50: R45-R61.

[2] Madsen M T, J Nucl Med, 2007, 48: 661-673

[3] Meikle S R, Kench P, Wojcik R, et al. Nuclear Science Symposium Conference Record, 2003, 3: 1988-1992

[4] Furenlid L R, Wilson D W, Chen Y C et al. IEEE Trans. Nucl. Sci., 2004, 51: 631-635

[5] Figueroa S D, Winkelmann C T, Volkert W A, et al. Nuclear Science Symposium Conference Record, 2005, 3: 1752-1756

[6] Weisenberger A G, Kross B, Majewski S, et al. IEEE Trans. Nucl. Sci., 1998, 45: 3053-3058

[7] Wojcik R, Majewski S, Steinbach D et al. IEEE Trans. Nucl. Sci., 1998, 45: 487-491.

[8] Pani R, Soluri A, Scafe R et al. IEEE Trans. Nucl. Sci., 1999, 46: 702-708.

[9] McElroy D P, MacDonald L R, Beekman F J et al. IEEE Trans. Nucl. Sci. 2002, 49: 2139-2147.

[10] Qi Y J, Tsui, B M W, Wang Y. IEEE NSS-MIC Conference Record, 2003, 4: 2325- 2329.

[11] Giokaris N D, Loudos G K, Maintas D et al, Nuclear Instruments and Methods in Physics Research, 2003, A 497: 141-149

[12] Bradley E.L, Cella J, Majewski S, et al , IEEE Trans. Nucl. Sci., 2006, 53: 59-65

[13] Seo H K, Choi Y, Kim, J H, et al. Nuclear Science Symposium Conference Record, 2000 ,3: $94-97$

[14] Schramm N, Wirrwar A, Sonnenberg F et al. IEEE Trans. Nucl. Sci., 2000, 47: 1163-1167.

[15] Zeng HaiNing, Xu ZiZong, Wang ZhaoMin et al, high energy physics and nuclear physics,2000, 24: 166-171

[16] Anger H O, Rev Sci \& Instr, 1958, 29(1): 27-33

[17] Wojcik R, Majewski S, Kross B et al., IEEE NSS-MIC Conference Record, 2001, 3: 1821-1825.

[18] Soluri A, Massari R, Trotta C, et al, Nuclear Instruments and Methods in Physics Research A 554 (2005) 331-339

[19] Popov V, Majewski S, Weisenberger A G, IEEE Nucl. Sci. Symp. Conf. Rec., 2003, 3:2156-2159. 